\title{
Effectiveness of task-shifting for the detection of diabetic retinopathy in low- and middle-income countries: a rapid review protocol
}

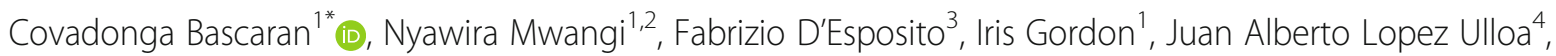
Shaffi Mdala ${ }^{5}$, Jacqueline Ramke ${ }^{1,6}$, Jennifer R. Evans ${ }^{1}$ and Matthew Burton ${ }^{1,7}$

\begin{abstract}
Background: Diabetic retinopathy is the most common ocular complication of diabetes and a cause of vision loss in adults. Diabetic retinopathy screening leading to early identification of the disease followed by timely treatment, can prevent vision loss in people living with diabetes. A key barrier to the implementation of screening services in low- and middle-income countries is the low number of ophthalmologists per million population. Interventions that shift screening to non-ophthalmology cadres have been implemented in programmes in low- and middle-income countries and are routinely used in high-income countries. The aim of this rapid review is to summarise the published literature reporting the effectiveness of task-shifting interventions for the detection of diabetic retinopathy by non-ophthalmologists in low- and middle-income countries.
\end{abstract}

Methods: We will search MEDLINE, Embase, Global Health and Cochrane Register of Studies for studies reporting task-shifting interventions for diabetic retinopathy detection. The review will include studies published in the last 10 years in the English language. We will include any interventional or observational comparative study measuring outcomes in terms of participation or access to diabetic retinopathy detection services (uptake) and quality of diabetic retinopathy detection services (detection, severity, diagnostic accuracy). For included studies, cost-effectiveness of the task-shifting intervention will also be presented. Two reviewers will screen search results independently. The risk of bias assessment and data extraction will be carried out by one reviewer with verification of $10 \%$ of the papers by a second reviewer. The results will be synthesised narratively.

Discussion: Differences in health systems organization, structure and resources will determine the need and success of task-shifting interventions for DR screening. The review will examine how these interventions have been used and/or tested in LMICs. The results will be of interest to policy makers and programme managers tasked with designing and implementing services to prevent and manage diabetes and its complications in similar settings.

(Continued on next page)

\footnotetext{
* Correspondence: Covadonga.bascaran@lshtm.ac.uk

${ }^{1}$ London School of Hygiene \& Tropical Medicine, Keppel Street, London

WC1E 7HT, UK

Full list of author information is available at the end of the article
}

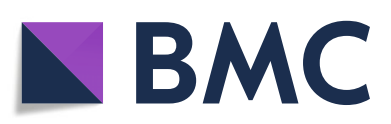

(c) The Author(s). 2021 Open Access This article is licensed under a Creative Commons Attribution 4.0 International License, which permits use, sharing, adaptation, distribution and reproduction in any medium or format, as long as you give appropriate credit to the original author(s) and the source, provide a link to the Creative Commons licence, and indicate if changes were made. The images or other third party material in this article are included in the article's Creative Commons licence, unless indicated otherwise in a credit line to the material. If material is not included in the article's Creative Commons licence and your intended use is not permitted by statutory regulation or exceeds the permitted use, you will need to obtain permission directly from the copyright holder. To view a copy of this licence, visit http://creativecommons.org/licenses/by/4.0/ The Creative Commons Public Domain Dedication waiver (http://creativecommons.org/publicdomain/zero/1.0/) applies to the data made available in this article, unless otherwise stated in a credit line to the data. 
(Continued from previous page)

Systematic review registration: OSF: https://osf.io/dfhg6/.

Keywords: Low- and middle-income countries, Diabetic retinopathy, Task-shifting, Effectiveness

\section{Background}

Diabetic retinopathy (DR) is the most frequent microvascular complication of diabetes and a significant cause of vision loss in adults [1]. Vision loss due to DR can largely be prevented through regular retinal screening for timely identification of DR, followed by referral and treatment [2-5].

DR screening has been shown to be cost-effective when compared to no screening or opportunistic screening $[3,6,7]$. However, cost-effectiveness requires high coverage and this is challenging in low- and middleincome (LMICs) countries [8] where the available eye health services are clustered around the cities and access to them from the remote and rural populations is often difficult [9]. Few countries worldwide have been successful in establishing population-based screening programmes that reach most of the patients with diabetes [3]. The uptake of retinal screening depends on contextspecific factors related to the service provider, the wider organization and structure of the health system and the patient [10-12].

A crucial limitation to deliver DR screening in LMICs is the low availability of ophthalmologists, particularly in sub-Saharan Africa (SSA), where the mean ophthalmologist density per million population is 3.7 [13]. In this context, it is not realistic to rely on ophthalmologists to examine the retinas of the growing population of people living with diabetes (PLWD) unless it is at the expense of the delivery of other critical eye care services. This is not only a challenge for LMICs, and programmes in high-income countries (HICs) have overcome this issue by successfully adopting task-shifting in DR screening by training retinal graders, thus freeing ophthalmologists time to perform other tasks [14]. The WHO defines task-shifting as the transfer of tasks to existing cadres of healthcare workers with shorter training and fewer qualifications or to newly created cadres who receive a competency-based training for the specific task [15]. There is some evidence of the successful use of taskshifting in SSA in other health fields including diabetes $[16,17]$. However, it is not clear whether task-shifting interventions for the detection of DR in LMICs results in an increase in the uptake of screening and higher DR detection rate.

There are several factors that can potentially affect the effectiveness of task-shifting interventions for DR screening. Firstly, the choice, level and training of the cadre performing the screening is a key consideration and is likely to be different depending on the composition and competencies of the health workforce in a particular country. The quality of the screening is measured by the diagnostic test accuracy (DTA) of the method of choice in the hands of non-ophthalmologists [18]. It is recommended that population-based screening programmes should reach over $80 \%$ sensitivity and $95 \%$ specificity $[6,19]$. Screening performed by photographers has been shown to be more accurate when done by photographers with specialist medical or eye care qualifications [20]. There is also evidence that non-medical retinal image graders can achieve the threshold level of sensitivity and specificity in both mydriatic and nonmydriatic retinal assessment [18].

Secondly, the screening technique used can also affect the DTA. The recommended technique is photographybased screening which adds the possibility of quality assurance [14]. DR screening programs in LMICs are gradually adopting retinal photography. However, in many clinics without cameras, other methods of retinal examination that are not necessarily effective as screening techniques are still used [18]. The sensitivity of direct ophthalmoscopy in detecting DR is low (65\%) and indirect ophthalmoscopy requires extensive training and experience [21]. Slit lamp bio-microscopy has a high sensitivity and specificity but also requires significant training and the availability of a slit lamp. Optical coherence tomography (OCT) for the detection of diabetic maculopathy is expensive and rarely available outside tertiary level eye departments in LMICs.

Thirdly, the use of mydriasis can improve the effectiveness of task-shifting by increasing the number of gradable images [18]. However, non-mydriatic imaging presents several advantages for screening programmes, like reduced screening time, less inconvenience for patients and importantly no limitations for using cadres that may not be allowed to instil eye drops.

Finally, an important consideration in LMICS is the need to reach rural and remote populations with difficult access to eye health services. Outreach services delivered by non-ophthalmologists can be an answer to this problem, but must ensure that DTA is maintained $[18,20]$. Evidence suggests that DTA is higher in hospital-based screening services compared with outreach services [15]. Several approaches for DR screening in outreach services that can lend themselves to task-shifting have shown promising accuracy levels. These include telemedicine using digital imaging, the use of artificial intelligence- 
supported cameras or smart phone-based technology for image capture [22-26].

This rapid review aims to identify and synthesise the peer reviewed literature on the effectiveness of taskshifting for the detection of DR in LMICs. It is an important topic given the predicted epidemic of diabetes mellitus which will disproportionally affect LMICs which will require major prevention strategies to prevent the corresponding increase in blindness caused by diabetic retinopathy. There is a body of evidence to support the effectiveness of task-shifting in DR screening in highincome settings which suggests that this may be a viable strategy in LMICs. However, given the differences in structure, organization and resourcing in health systems in LMICs, evidence from HICs may not be generalizable to these settings. The aim of this rapid review is to summarise the published literature reporting the effectiveness of task-shifting interventions for the detection of diabetic retinopathy by non-ophthalmologists low- and middle-income countries.

\section{Methods}

\section{Rapid review question}

Can task-shifting improve the detection of diabetic retinopathy (DR) compared with eye examination done by ophthalmologists among adults with diabetes in LMICs?

\section{Protocol and registration}

A rapid review was chosen as it is an effective methodology to support health programme decision-making through quality evidence in a timely and cost-effective manner. These rapid review methods were informed by the WHO practical guide on rapid reviews and we used the STARR (Selecting Approaches for Rapid Reviews) Decision Tool to carefully take into account the possible limitations introduced by the choice of methodology [27, 28]. We provide the adapted Preferred Reporting Items for Systematic Reviews and Meta-Analysis Protocols (PRISMA-P) in Additional file 1. The registration details are provided in Additional file 2. We will document any future amendments to the protocol on the Open Science Framework (OSF) registration site [29] (Table 1).

\section{Eligibility criteria}

The review will focus on adults only as they represent the larger population group at risk of DR and are the target of population-based screening programmes. We will include any study that measures any of the uptake and quality outcomes described in the above table. For included studies, we will also collect data on cost-effectiveness of the taskshifting intervention where available.

We will only include peer-reviewed publications reporting studies conducted in LMICs, as classified by
Table 1 Eligibility criteria

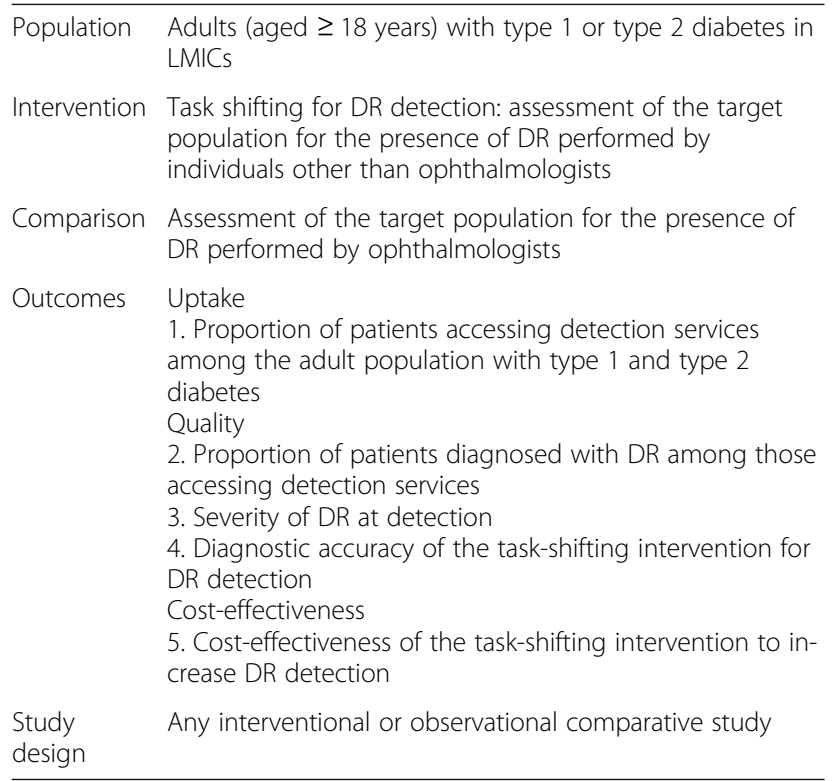

the World Bank [8] in the last 10 years, in the English language with available full reports.

The following exclusion criteria will be applied: firstly, reviews, editorials, case studies, conference abstracts; secondly, studies involving health education, training programmes or qualitative studies and thirdly, studies conducted in high income settings.

\section{Information sources}

Following guideline of the STARR Decision tool, we consulted the review commissioners (FHF) and conducted scoping searches on PubMed to inform the decision on which information sources to include, balancing the scope and time available to do the review [30]. The Cochrane Eyes and Vision Information Specialist (IG) will develop the search strategy which will include MEDLINE, Embase, Global Health and the Cochrane Register of Studies. We will not search the grey literature as it was considered that the priority for this review is to summarise the peer-reviewed evidence of effectiveness of task-shifting interventions required to influence and change policy and practice.

\section{Search strategy}

Search strategy is included in Additional file 3.

\section{Data management and selection process}

Two reviewers will independently review each title and abstract using the online review management software (Covidence, Veritas Health Innovation, Melbourne, Australia. Available at www.covidence.org). Any conflicts will be discussed and resolved. Two reviewers will independently screen the full text articles of potentially 
relevant studies against the inclusion and exclusion criteria and any will discuss any differences to reach consensus. A summary of the study selection process will be presented in a PRISMA flow diagram.

\section{Risk of bias assessment}

One reviewer will use SING critical appraisal checklists (https://www.sign.ac.uk) to assess the risk of bias for included studies. This will be presented in narrative form with the review findings.

\section{Data extraction}

We will develop an Excel data extraction form which will be tested by two reviewers on three papers. One reviewer will conduct data extraction and a second

Table 2 Data items

Data
categories

reviewer will check $10 \%$ of the studies for accuracy. If more than $1 \%$ errors are found, another $10 \%$ will be checked (Table 2).

\section{Data synthesis}

As we anticipate to find heterogeneity in study design, interventions, methods and outcome reporting, we will summarise data narratively following the SWiM reporting guidance: Synthesis Without Meta-analysis reporting items [31]. We will use tables to present the scope and nature of the evidence and descriptive statistics and visual displays to present effect estimates. We will use the GRADE (Grading of Recommendations, Assessment, Development and Evaluations) approach to assess the certainty of the evidence for the outcomes of the review and report any limitations of the synthesis and how they may affect the conclusions.

\section{Discussion}

Differences in health systems organization, structure and resources will determine the need and success of taskshifting interventions for DR screening. The review will examine how these interventions have been used and/or tested in LMICs. This rapid review will provide contextspecific evidence to inform policy makers and programme managers tasked with designing and implementing services to prevent and manage diabetes and its complications in low- and middle- income settings.

In balancing the time demands of a rapid review methodology, we have prioritized the peer-reviewed literature as we considered the more robust evidence for effectiveness will be found in published scientific journals. We acknowledge that the limits in searches and time may potentially miss some evidence which could have been published before the period included and in the grey literature.

\section{Supplementary Information}

The online version contains supplementary material available at https://doi. org/10.1186/s13643-020-01553-w.

Additional file 1. Reporting standards - PRISMA-P Checklist.

Additional file 2. Registration OSF: https://osf.io/h5wgr/

Additional file 3. Search strategy.

\footnotetext{
Abbreviations

DR: Diabetic retinopathy; LMIC: Low- and middle-income country; SSA: SubSaharan Africa; PLWD: People living with diabetes; HIC: High-income country; WHO: World Health Organization; DTA: Diagnostic test accuracy; OCT: Ocular coherence tomography; OSF: Open Science Framework; PRISMA-P: Preferred Reporting Items for Systematic Reviews and Meta-Analysis Protocols; SING: Scottish Intercollegiate Guidelines Network; EDTRS: Early Treatment Diabetic Retinopathy Study; CPD: Continuing professional development; SWiM: Synthesis without meta-analysis; GRADE: Grading of Recommendations Assessment, Development and Evaluation
} 


\section{Acknowledgments}

Not applicable.

\section{Authors' contributions}

FD conceived the idea for the review. CB and NM drafted and revised the protocol with suggestions from JE, JR, FD, SM, JALU, and MJB who reviewed the protocol and provided feedback on the draft. IG constructed the search. The authors read and approved the final manuscript.

\section{Funding}

This review was commissioned and funded by Fred Hollows Foundation (FHF) Australia. MJB is supported by the Wellcome Trust (207472/Z/17/Z)

\section{Availability of data and materials}

Not applicable — no data sets currently available.

Ethics approval and consent to participate

Not applicable.

\section{Consent for publication}

Not applicable.

\section{Competing interests}

None declared.

\section{Author details}

'London School of Hygiene \& Tropical Medicine, Keppel Street, London WC1E 7HT, UK. ${ }^{2}$ Kenya Medical Training College, Nairobi, Kenya. ${ }^{3}$ The Fred Hollows Foundation, Melbourne, Australia. ${ }^{4}$ Centro Mexicano de Salud Visual Preventiva, Mexico, DF, Mexico. ${ }^{5}$ Queen Elizabeth Central Hospital, Blantyre, Malawi. ${ }^{6}$ School of Optometry and Vision Science, University of Auckland, Auckland, New Zealand. ${ }^{7}$ Moorfields Eye Hospital, London, UK.

Received: 14 May 2020 Accepted: 2 December 2020

Published online: 04 January 2021

\section{References}

1. Klein BE. Overview of epidemiologic studies of diabetic retinopathy Ophthalmic Epidemiol. 2007;14(4):179-83.

2. Association AD. Improving care and promoting health in populations: standards of medical care in diabetes-2020. Diabetes Care. 2020;43(Suppl 1): S7-s13.

3. Swanson M. Retinopathy screening in individuals with type 2 diabetes: who, how, how often, and at what cost-an epidemiologic review. Optometry. 2005;76(11):636-46.

4. Cheung N, Mitchell P, Wong TY. Diabetic retinopathy. Lancet. 2010; 376(9735):124-36

5. Stewart MW. Treatment of diabetic retinopathy: recent advances and unresolved challenges. World J Diabetes. 2016;7(16):333-41.

6. Mead A, Burnett S, Davey C. Diabetic retinal screening in the UK. J R Soc Med. 2001;94(3):127-9.

7. Jones S, Edwards RT. Diabetic retinopathy screening: a systematic review of the economic evidence. Diabet Med. 2010;27(3):249-56.

8. Bank W World Bank country and lending groups; 2020. [cited 2020 July]. Available from: https://datahelpdesk.worldbank.org/knowledgebase/articles/ 906519-world-bank-country-and-lending-groups.

9. Vashist P, Singh S, Gupta N, Saxena R. Role of early screening for diabetic retinopathy in patients with diabetes mellitus: an overview. Indian J Community Med. 2011:36(4):247-52

10. Cavan D. The diabetes epidemic and its implications for eye health. Community Eye Health. 2015;28(92):61-3.

11. Lewis K. Improving patient compliance with diabetic retinopathy screening and treatment. Community Eye Health. 2015;28(92):68-9.

12. Cavan D, Makaroff L, da Rocha FJ, Sylvanowicz M, Ackland P, Conlon J, et al. The diabetic retinopathy barometer study: global perspectives on access to and experiences of diabetic retinopathy screening and treatment. Diabetes Res Clin Pract. 2017;129:16-24.

13. Resnikoff S, Lansingh VC, Washburn L, Felch W, Gauthier TM, Taylor HR, et al. Estimated number of ophthalmologists worldwide (International Council of Ophthalmology update): will we meet the needs? Br J Ophthalmol. 2020; 104(4):588-92
14. Scanlon PH. The English National Screening Programme for diabetic retinopathy 2003-2016. Acta Diabetol. 2017:54(6):515-25.

15. WHO. World Health Organization. Task shifting: global recommendations and guidelines. Geneva: World Health Organization; 2008

16. Ong SE, Koh JJK, Toh SES, Chia KS, Balabanova D, McKee M, et al. Assessing the influence of health systems on type 2 diabetes mellitus awareness, treatment, adherence, and control: a systematic review. PLoS One. 2018; 13(3):e0195086.

17. Nuche-Berenguer B, Kupfer LE. Readiness of sub-Saharan Africa healthcare systems for the new pandemic, diabetes: a systematic review. J Diabetes Res. 2018:2018:9262395.

18. Piyasena M, Murthy GVS, Yip JLY, Gilbert C, Peto T, Gordon I, et al. Systematic review and meta-analysis of diagnostic accuracy of detection of any level of diabetic retinopathy using digital retinal imaging. Syst Rev. 2018;7(1):182.

19. Scanlon PH, Aldington SJ, Leal J, Luengo-Fernandez R, Oke J, Sivaprasad S, et al. Development of a cost-effectiveness model for optimisation of the screening interval in diabetic retinopathy screening. Health Technol Assess. 2015;19(74):1-116.

20. Bragge P, Gruen RL, Chau M, Forbes A, Taylor HR. Screening for presence or absence of diabetic retinopathy: a meta-analysis. Arch Ophthalmol. 2011; 129(4):435-44.

21. Benbassat J, Polak BC, Javitt JC. Objectives of teaching direct ophthalmoscopy to medical students. Acta Ophthalmol. 2012;90(6):503-7.

22. Bilong Y, Katte JC, Koki G, Kagmeni G, Obama OPN, Fofe HRN, et al. Validation of smartphone-based retinal photography for diabetic retinopathy screening. Ophthalmic Surg Lasers Imaging Retina. 2019;50(5): S18-s22.

23. Sabanayagam C, Yip W, Ting DS, Tan G, Wong TY. Ten emerging trends in the epidemiology of diabetic retinopathy. Ophthalmic Epidemiol. 2016;23(4):209-22

24. Shi L, Wu H, Dong J, Jiang K, Lu X, Shi J. Telemedicine for detecting diabetic retinopathy: a systematic review and meta-analysis. Br J Ophthalmol. 2015; 99(6):823-31.

25. Hansen MB, Abramoff MD, Folk JC, Mathenge W, Bastawrous A, Peto T. Results of automated retinal image analysis for detection of diabetic retinopathy from the Nakuru study, Kenya. PLoS One. 2015;10(10):e0139148.

26. Bellemo V, Lim G, Rim TH, Tan GSW, Cheung CY, Sadda S, et al. Artificial intelligence screening for diabetic retinopathy: the real-world emerging application. Curr Diab Rep. 2019;19(9):72.

27. Cochrane. Rapid Reviews Methods Group [cited 202021 February]. Available from: https://methods.cochrane.org/news/rapid-reviews-methods-group.

28. PRISMA. Preferred Reporting Items for Systemactic Reviews and Meta-Analyses [21 February 2020]. Available from: http://www.prisma-statement.org/.

29. Bascaran C, Mwangi N, D'Esposito F, Mdala S, Ulloa JAL, Gordon I, et al. Effectiveness of task-shifting for the detection of diabetic retinopathy in low- and middle-income countries: a rapid review protocol; 2020.

30. Pandor AKE, Martyn-St James M, Wong R, Cooper K, Dimairo M, O'Cathain A, et al. Delphi consensus reached to produce a decision tool for SelecTing approaches for rapid reviews (STARR). J Clin Epidemiol. 2019;114:22-9..

31. Campbell M, McKenzie JE, Sowden A, Katikireddi SV, Brennan SE, Ellis S, et al. Synthesis without meta-analysis (SWiM) in systematic reviews: reporting guideline. BMJ (Clinical research ed). 2020;368:16890.

\section{Publisher's Note}

Springer Nature remains neutral with regard to jurisdictional claims in published maps and institutional affiliations.

Ready to submit your research? Choose BMC and benefit from:

- fast, convenient online submission

- thorough peer review by experienced researchers in your field

- rapid publication on acceptance

- support for research data, including large and complex data types

- gold Open Access which fosters wider collaboration and increased citations

- maximum visibility for your research: over $100 \mathrm{M}$ website views per year

At $\mathrm{BMC}$, research is always in progress.

Learn more biomedcentral.com/submission 